\title{
APPLICATION OF $\alpha$-ADRENOMIMETIC DECONGESTANTS AS CHEMICAL AGENTS FOR GINGIVAL RETRACTION
}

\author{
Iveta Katreva ${ }^{1}$, Metodi Abadjiev ${ }^{1}$, Simeon Simeonov', Ognjan Tivchev ${ }^{1}$, Maya Doychinova ${ }^{2}$, \\ Tsvetan Tonchev ${ }^{3}$ \\ ${ }^{1}$ Department of Prosthetic Dentistry, Faculty of Dental Medicine, \\ Medical University of Varna \\ ${ }^{2}$ Department of Conservative Dentistry, Faculty of Dental Medicine, \\ Medical University of Varna \\ ${ }^{3}$ Department of Maxillofacial Surgery, Faculty of Dental Medicine, \\ Medical University of Varna
} ABSTRACT

Purpose: The purpose of this study is to observe, compare and evaluate the effect of two $\alpha$-adrenomimetic decongestants which are clinically approved nasal and eye drops $\mathbf{- 0 . 0 5 \%}$ xylometazoline hydrochloride and $\mathbf{0 . 0 5 \%}$ tetrahydrozoline hydrochloride, when applied as chemical agents for chemo-mechanical retraction of free gingiva prior to impression making in fixed prosthodontics.

Materials and methods: The study includes 90 prepared abutment teeth indicated for fixed prosthodontic treatment. $a$-adrenomimetic decongestants in the composition of 2 approved for clinical usage nasal and eye drops commercial products were applied as chemical agents for gingival retraction - Xylometazoline ( $0.05 \%$ xylometazoline hydrochloride) and Visine ( $0.05 \%$ tetrahydrozoline hydrochloride). Ultrapak retraction cord of four different sizes impregnated with the investigated chemical agents was used. Two steps twolayered impressions with polyvinyl and polydimethylsiloxane were taken from the prosthetic fields. Impression sections were fabricated and studied under microscope.

Results: The low viscosity polyvinyl and polydimethyl siloxane layers tend to penetrate deeper in the gingival groove after chemo-mechanical retraction with Xylometazoline compared to the second group impressions fabricated after Visine retraction.

Conclusion: The conducted testing demonstrated that effective retraction of the free marginal gingiva is possible to obtain with $a$-adernomimetic decongestants. $0.05 \%$ xylometazoline hydrochloride (Xylometazoline) provides better results in comparison to the eye decongestant drops $0.05 \%$ tetrahydrozoline hydrochloride (Visine).

Keywords: gingival retraction, retraction agents, chemo-mechanical retraction

Address for correspondence:

Iveta Katreva

Department of Prosthetic Dentistry

Faculty of Dental Medicine

Medical University of Varna

55 Marin Drinov Str.

9002 Varna, Bulgaria

e-mail:ikatreva@yahoo.com

Received: August 7, 2015

Accepted: December 1, 2015

\section{INTRODUCTION}

To carry through a contemporary treatment plan in accordance to the functional, prophylactic and esthetic requirements in fixed prosthodontics the dental specialist needs highly precise impressions for perfect marginal fit of the constructions. Gingival retraction involves the displacement of marginal soft tissues around a tooth, mainly to allow access in preparation, precise impression taking and cementa-

Scripta Scientifica Medicinae Dentalis, vol. 1, №2, 2015, pp. 17-23 
tion procedures. It is often needed for subgingival direct restorations and manipulations in the cervical tooth area in conservative dentistry too. The optical impressions need even wider vertical and horizontal displacement of margo gingivalis (5).

One of the main problems in impression making is how to assure the access of the low viscosity impression materials not only to but also beyond the preparation shoulder especially when it is located below the gingiva for esthetic reasons. This is the critical moment for the successful and exact reproduction of the marginal details over the working cast and for the fabrication of high quality constructions (4). The presence of blood and crevice fluid in the gingival groove are also stumbling stones for the impression procedures.

There is a big variety of commercial products and methods to obtain temporary dilatation of the sulcus gingivalis. The choice of the clinicians is usually based upon the personal preferences and according to the individual case $(1,8,11)$.

One of the most frequently used methods is chemo-mechanical retraction via cord, impregnated with a chemical agent to obtain hemostasis and reduction of crevice fluid. Hansen et al report that $98 \%$ of the prosthetic specialists use retraction cord, $48 \%$ prefer "double-cord" technique and $44 \%$ insert single cord into gingival sulcus (10).

A study by Abadzhiev proves the advantages of "double-cord" retraction protocol and names it a "standard" in prosthetic dentistry (2).

According to some scientific researches there are 125 types of retraction cords available on the dental market (3).

They differ in size, filament arrangement and impregnation (18).

Sympathicomimetic (vasoconstrictors) and astringents (clotting agents) are the 2 main groups of impregnating chemical substances (9) They could be applied in various concentration in gel, liquid or paste consistency. Among sympathicomimetic agents, epinephrine (adrenalin) is preferred for its effective vasoconstriction over the peripheral blood vessels in the mucosa and relevantly good hemostasis. Even applied topically in gingival sulcus, adrenalin causes systematic side effects through $\beta-1$ and $\beta-2$ adrenergic receptors and affects the overall health of the patient (7). Systematic side effects as tachycardia, arrhythmia, collapse, increased respiratory and pulse rates, paleness, nervousness, postoperative depression are reported. The condition is known as "epinephrine syndrome" $(6,17)$. Adrenalin is contraindicated in cases of heart or endocrine diseases. On the other hand, there are enough scientific proofs about the strong cytotoxic effect of the epinephrine impregnated retraction cords over the gingival fibroblasts even in short exposure periods (13).

The conventional retraction agents from the group of astringents are metal salts in different concentration - aluminium chloride, aluminium sulfate, ferric sulfate, zinc chloride, etc. A lot of in vitro experiments demonstrate their harmful effect not only on gingival tissues but also hard tooth tissues $(12,13,14)$. Aluminum sulfate shows the lowest cytotoxicity, followed by aluminum chloride and ferric sulfate as the most harmful agent (15). There is scientific data that $25 \%$ aluminum chloride damages completely the cell cultures of gingival fibroblasts in only 1 minute exposure period (12) (Fig. 1, Fig. 2).

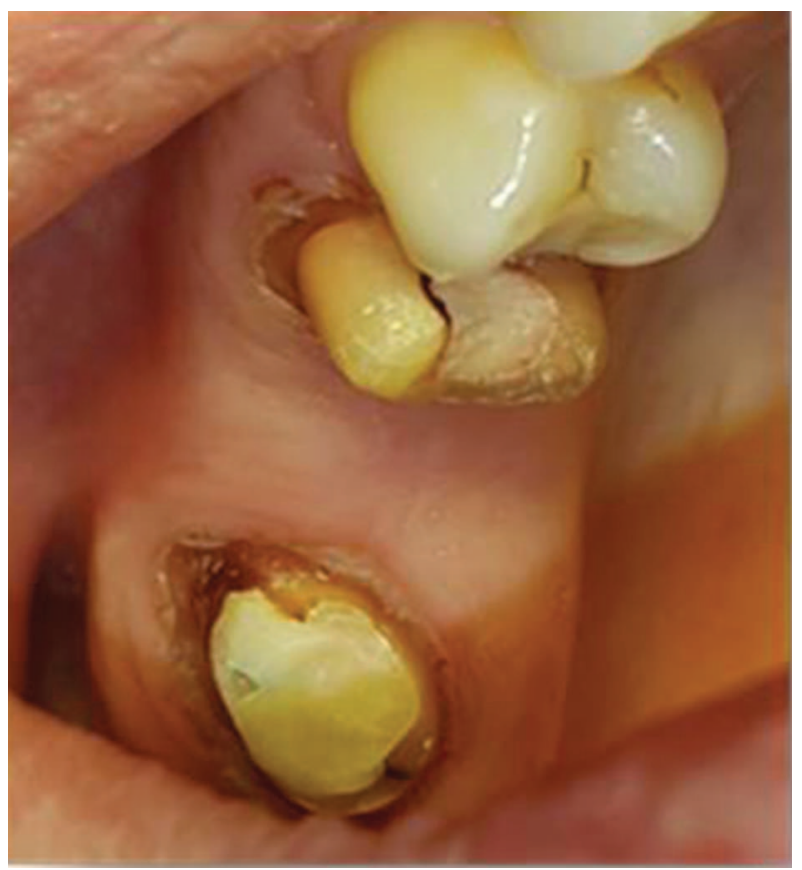

Figure 1. Clinical appearance of the free gingiva after chemo-mechanical retraction with $\mathrm{Al}_{2} \mathrm{Cl}_{3}-2^{\text {nd }}$ day

Moreover, the astringent compounds are chemically stable and active only within a narrow diapason of acidic $\mathrm{pH}$ which causes etching of the hard 


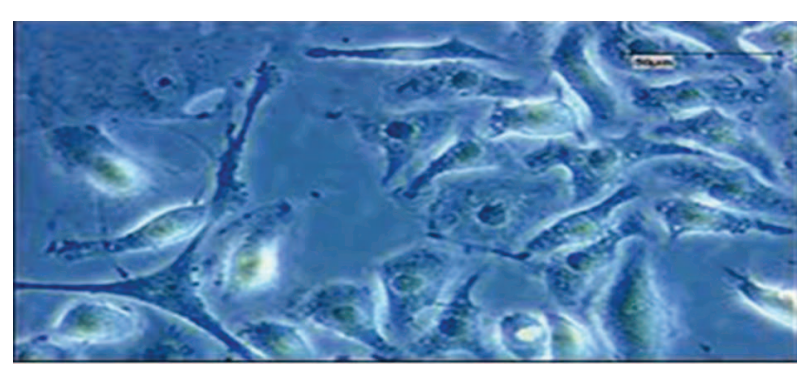

Figure 2. Electronic microscopic picture of human gingival fibroblasts

tooth tissues and postoperative sensitivity of vital teeth (11).

The mentioned above disadvantages of conventional retraction agents provoke scientific studies to continue searching for the most biologically compatible chemical compounds for cord impregnation. Bowles et al brought to light a new application for the nasal and eye decongestants oxymetazoline hydrochloride, xylometazoline hydrochloride and tetryzoline. They studied the effect of 3 commercial products - Visine ${ }^{\circledast}$ (tetrahydrozoline $\mathrm{HCl} 0.05 \%$, Pfizer, Warszawa, Poland) (Fig. 3), Afrin (oxymetazoline 0.05\%, Schering- Plough, Brussels, Belgium) (Fig. 4) and Neosynephrine ${ }^{\circledast}$ (phenylephrine $\mathrm{HCl} 0.25 \%$, Ursapharm, Saarbrücken, Germany) (Fig. 5).

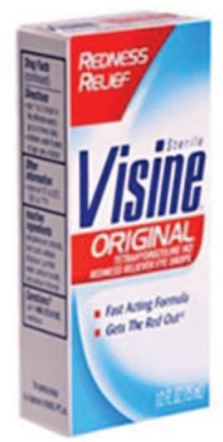

Figure 3. Visine (tetrahydrozoline $\mathrm{HCl}$ 0,05\%, Pfizer, Warszawa, Poland)

The conclusion is that Visine shows the best ability for hemostasis and tissue retraction and all 3 $\alpha$-adrenomimetic compounds lead to only a slight increase of arterial blood pressure (6). Later researches demonstrate through colorimetric tests of the mitochondrial oxidoreductive potential that the vitality of the gingival fibroblast cell cultures in vitro

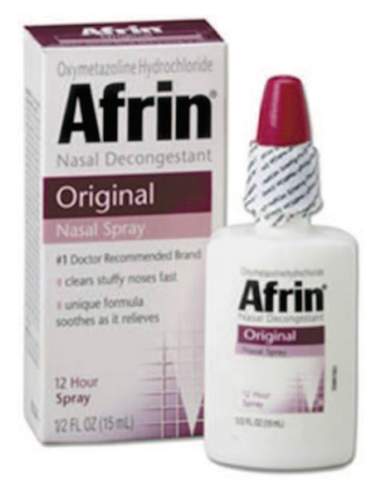

Figure 4. Afrin (oxymetazoline 0,05\%, Schering-Plough, Brussels, Belgium)

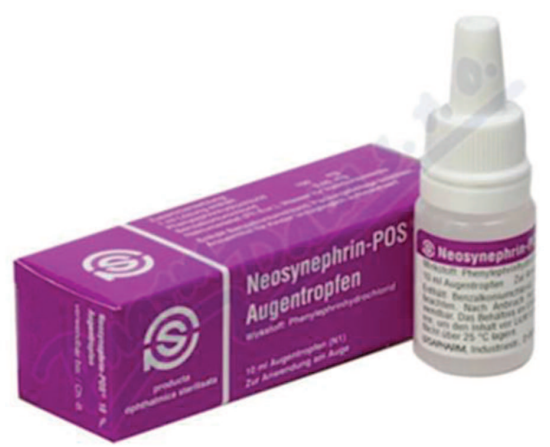

Figure 5. Neosynephrine (phenylephrine $\mathrm{HCl}$ 0,25\%, Ursapharm, Saarbrücken, Germany

treated with $0.05 \% \mathrm{HCl}$-tetrahydrozoline does not decrease under $50 \%$ in all exposure periods $-3,5,10$ min and even 24 hours (16). The 3 mentioned above $\alpha$-adrenomimetic compounds lead to effective retraction of margo gingivalis without affecting the periodontal and overall health.

Although Bowles et al reported promising data about effectiveness of $\alpha$-adrenomimetic substances as retraction agents in 1991, they still are called "experimental" in the science literature.

\section{MATERIALS AND METHODS}

The present study observes, compares and evaluates the effect of two $a$-adrenomimetic decongestants which are clinically approved nasal and eye drops - $0.05 \%$ xylometazoline hydrochloride and $0.05 \%$ tetrahydrozoline hydrochloride, when applied as chemical agents for chemo-mechanical retraction of free gingiva prior to impression taking in fixed prosthodontics. 
Application of $\alpha$-adrenomimetic decongestants as chemical agents for gingival retraction

Table 1. Some $\alpha$-adrenomimetic decongestants available as nasal and eye drops. Products suitable for retraction agents

\begin{tabular}{|c|c|c|c|}
\hline Brand name & Producer & Type & Active substance \\
\hline Visine ${ }^{\bowtie}$ Classic & Pfizer, Warszawa, Poland & $\begin{array}{l}\text { Ophthalmic decongestant, } \\
\text { drops }\end{array}$ & $\begin{array}{l}0,05 \% \text { tetrahydrozoline } \\
\mathrm{HCl}\end{array}$ \\
\hline Starazolin ${ }^{\circledast}$ & Pfizer, Warszawa, Poland & $\begin{array}{l}\text { Ophthalmic decongestant, } \\
\text { drops }\end{array}$ & $\begin{array}{l}0,05 \% \text { tetrahydrozoline } \\
\mathrm{HCl}\end{array}$ \\
\hline $\begin{array}{l}\text { Neosynephrine } \\
\text { POS }^{\circledast} 10 \%{ }^{\star}\end{array}$ & $\begin{array}{l}\text { Ursapharm, Saarbrücken, } \\
\text { Germany }\end{array}$ & $\begin{array}{l}\text { Ophthalmic decongestant, } \\
\text { drops }\end{array}$ & phenylephrine $\mathrm{HCl} 10 \%$ \\
\hline Afrin ${ }^{\oplus}$ & $\begin{array}{l}\text { Schering- Plough, Brussels, } \\
\text { Belgium }\end{array}$ & $\begin{array}{l}\text { Nasal decongestant, } \\
\text { Drops/spray }\end{array}$ & $0,05 \%$ oxymetazoline \\
\hline Xylometazolin 0,05\% & $\begin{array}{l}\text { Warsaw Pharmaceutical } \\
\text { Works Polfa S.A., Poland }\end{array}$ & $\begin{array}{l}\text { Nasal decongestant, } \\
\text { Drops }\end{array}$ & $\begin{array}{l}\text { Xylometazoline } \\
\text { hydrochloride } 0,05 \%\end{array}$ \\
\hline
\end{tabular}

${ }^{*}$ Neosynephrine POS $10 \%$ is not a registered product in Bulgaria

* 90 prepared teeth for fixed prosthetic constructions;

* Electronic periodontal probe Pa-on (Orangedental GmbH \& Co. KG, Germany)

* Ultrapak retraction cord (Ultradent Products Inc., USA) of 4 sizes $(000,00,0,1)$;

* Visine ${ }^{\circledast}$ Classic (tetrahydrozoline hydrochloride $0.05 \%$, Pfizer, Warszawa);

* Xylometazolin 0.05\% (Xylometazoline hydrochloride $0.05 \%$, Warsaw Pharmaceutical Works Polfa S.A., Poland);

* Polyvinylsiloxane impression material - Affinis Putty soft \& Affinis Precious regular body (Coltene /Whaledent Inc.);

* Polydimethylsiloxane impression material Zetaflow Hydrophilic Putty \& Zetaflow Hydrophilic Light (@Zhermack Clinical SpA, Italy);

* Standard metal perforated impression trays;

- Electronic microscope AmScope SM-5TZFOR-5M (AmScope Company, USA) (Fig. 6);

* Software - ZEN 2012 Blue Edition (Carl Zeiss Microscopy $\mathrm{GmbH}$ )



Figure 6. Electronic microscope AmScope SM-5TZ-FOR$5 M($ Amscope Company, USA)
The research started in 2012 and was performed on 46 patients with a total number of 90 prepared teeth. Measurements of the depth of gingival sulcus of each tooth were conducted with electronic periodontal probe $\mathrm{Pa}$-on (Orangedental $\mathrm{GmbH} \&$ Co. KG, Germany) in 4 points - mesio-vestibular, distovestibular, mediolingualis, distolingualis - (fig. 7). The data from the device had been simultaneously exported to the ByzzParo software (ver. 2.0.4) and stored in electronic periodontal diagrams, generated automatically. 360 measurements were thus conducted.

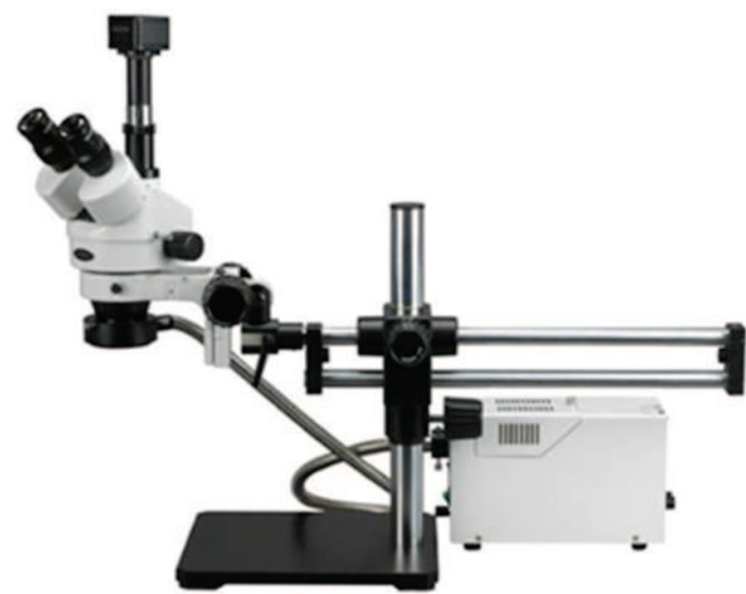

Figure 7. Electronic periodontal probe Pa-on (Orangedental GmbH \& Co. KG, Germany)

The teeth were prepared for metal-ceramic full crowns with preparation shoulder at gingival level. 
Iveta Katreva, Metodi Abadjiev, Simeon Simeonov et al.

The preparation of all teeth was performed by a single operator. Plain retraction cord (Ultrapak) was impregnated ex tempore by soaking into Visine ${ }^{\oplus}$ (tetrahydrozoline hydrochloride $0.05 \%$ ) or Xylometazolin $0.05 \%$ (Xylometazoline hydrochloride $0.05 \%$ ) for 20 minutes before insertion. Then, according to the chosen retraction agent and impression material, the preparations were divided into 2 groups.

\section{The $1^{\text {st }}$ group:}

The gingival sulcus of 45 teeth was retracted with Xylometazolin 0.05\% (Xylometazoline hydrochloride $0.05 \%$ ) impregnated cord. Then twostep double-layered full-arch impression technique with additional silicone (polyvinylsiloxane impression material - Affinis Putty soft \& Affinis Precious regular body) was used. After a period of 14 days a second retraction was performed with Visine $e^{ø}$ (tetrahydrozoline hydrochloride $0,05 \%$ ) and a second impression was taken with the same material and technique.

\section{The $2^{\text {nd }}$ group:}

It included also 45 prepared teeth. The retraction was carried out with Xylometazolin $0.05 \%$ at first and 2 weeks later cords impregnated with Visine were inserted to displace the free marginal gingiva. After each chemo-mechanical retraction a two-step double-layered full-arch impression technique with condensation silicone (polydimethylsiloxane impression material - Zetaflow Putty \& Zetaflow Light) was proceeded and sections fabricated.

In both groups the retraction cords were prepared under equal circumstances and the same size was used for the first and the second retraction of each tooth. The cords were left into gingival sulcus for 5 minutes before impression taking.

720 sections in the area of the electronic periodontal measurements were prepared from the impressions. All sections were studied under the electronic microscope AmScope SM-5TZ-FOR-5M with 35 magnification (35x) and frames were captured. The assessment of the retraction effect was implemented by measuring the length of the impression material's ingress into sulcus gingivalis on the microscopic pictures of the sections by the software program ZEN 2012 Blue Edition (Carl Zeiss Microscopy $\mathrm{GmbH}$ ).
Statistical analysis was performed using commercial SPSS v. 20 for Windows. The significance of differences between the mean values of the different groups was assessed by ANOVA analysis with values of $p<0.05$ taken to imply statistical significance.

\section{RESULTS}

The length of the low viscosity silicone ingress into the gingival sulcus of each of the 720 sections served to analyze and compare the retraction effect of the $2 \alpha$-adrenomimetic substances that had been applied. The data was systematically divided into two groups according to the impression material (Fig. 8, Fig. 9). Each group had two subcategories depending on the retraction agent.

The $1^{\text {st }}$ group included a total number of 360 sections from polyvinylsiloxane impressions (Fig. 8) of 45 prepared teeth. 180 of them were fabricated from the impressions taken after a retraction of the gingival sulcus of the teeth with Xylometazoline $(0.05 \%$ xylometazoline hydrochloride). The other $180 \mathrm{sec}-$ tions were made after Visine retraction $(0.05 \%$ tetrahydrozoline hydrochloride) and second impression taking of the same teeth 2 weeks later. The mean values of the variables in the two subcategories of the group are shown in the next table.

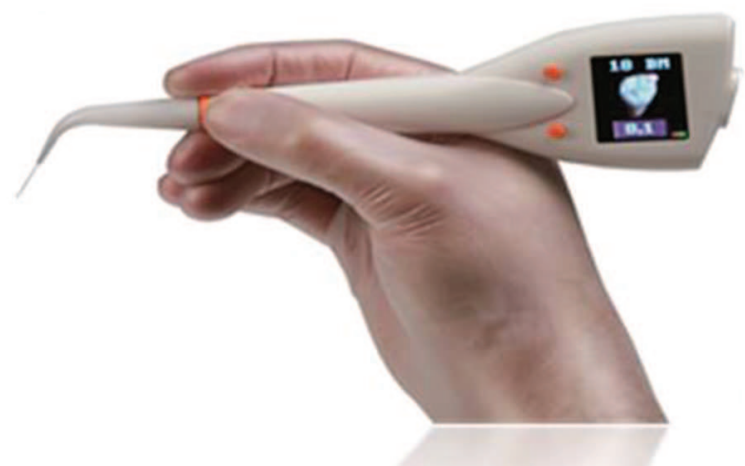

Figure 8. Sections prepared from polyvinylsiloxane impressions - images made by software - ZEN 2012 Blue Edition (Carl Zeiss Microscopy GmbH) under microscope (x35)

The $2^{\text {nd }}$ group is presented by 360 sections fabricated from polydimethylsiloxane impressions (Fig. 9) of 45 prepared teeth. 180 of the sections were fabricated from the impressions taken after the first retraction of the soft tissues around the preparations

Scripta Scientifica Medicinae Dentalis, vol. 1, №2, 2015, pp. 17-23 
Application of $\alpha$-adrenomimetic decongestants as chemical agents for gingival retraction

Table 2. Mean values and difference of the polyvinylsiloxane ingress into gingival sulcus after retraction with Xylometazoline and Visine

\begin{tabular}{|c|c|c|c|c|c|}
\hline & Xylometazoline & Visine & Difference & $\mathrm{F}$ & $\mathrm{p}$ \\
\hline Depth of gingival sulcus measured with $\mathrm{Pa}$-on (mm) & 1,57 & 1,57 & - & - & - \\
\hline $\begin{array}{l}\text { Ingress of Affinis Precious regular body layer of the } \\
\text { section after retraction }(\mathrm{mm})\end{array}$ & 0,84 & 0,66 & 0,18 & 36,63 & $<0,001$ \\
\hline Value of the ingress (in \%) & 57,23 & 44,45 & 12,78 & 41,32 & $<0,001$ \\
\hline
\end{tabular}

performed by $0.05 \%$ Xylometazolin. The second retraction of the same teeth was done after a period of 2 weeks with Visine impregnated cords. Thus another 180 sections were obtained for analysis. The mean values of the depth of the gingival sulcus, as well as the impression material ingress in $\mathrm{mm}$ and percents are presented in the table below (Table 3).
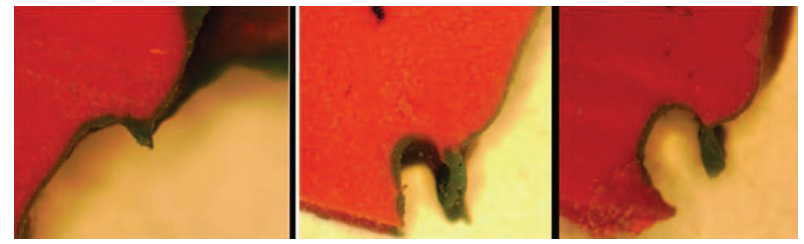

Figure 9. Sections from polydimethylsiloxane impressionsimages made by software - ZEN 2012 Blue Edition (Carl

Zeiss Microscopy GmbH) under microscope (x35) gingival groove after both kind of chemo-mechanical retractions in favor of Xylometazolin $(\mathrm{p}<0.001)$. The number of impression sections where the material has reached more than half of the sulcular depth is $17.80 \%$ more when Xylometazoline is applied. The results determine that $0.05 \%$ xylometazoline hydrochloride is a more effective impregnating agent for chemo-mechanical retraction of the free gingival margin.

For the $2^{\text {nd }}$ group impression sections of polydimethylsiloxane material (C-silicone) the mean value of the ingress after the application of Xylometazolin is $59.28 \% \pm 15.24 \%$ and $76.10 \%$ of these sections have low viscosity layer beyond the half of the sulcular depth. In the cases with Visine retrac-

Table 3. Mean values and difference of the polydimethylsiloxane ingress into gingival sulcus after retraction with Xylometazoline and Visine

\begin{tabular}{l|cc|c|c} 
& Xylometazoline & Visine & Difference & F \\
$\begin{array}{l}\text { Depth of gingival sulcus measured with Pa-on } \\
\text { (mm) }\end{array}$ & 1,83 & 1,83 & - & - \\
$\begin{array}{l}\text { Ingress of Zetaflow Hydrophilic Light } \\
\text { section after retraction (mm) }\end{array}$ & & & - \\
\begin{tabular}{l} 
Value of the ingress (in \%) \\
\hline
\end{tabular} & 59,28 & 47,37 & 11,91 & $54,04<0,001$ \\
\hline
\end{tabular}

\section{DISCUSSION}

The collected data from the $\mathbf{1}^{\text {st }}$ group of impression sections has shown $57.23 \% \pm 24.74 \%$ average ingress of the low viscosity polyvinylsiloxane impression material into the gingival sulcus after $0.05 \% \mathrm{Xy}$ lometzolin (0.05\% xylometazoline hydrochloride) retraction and $44.45 \% \pm 24.19 \%$ after the retraction with Visine ( $0.05 \%$ tetrahydrozoline hydrochloride). This is a significant difference of almost $13 \%$ between the access of the additional silicone into the tion agent the mean value of the impression ingress is $47.37 \% \pm 14.49 \%$ and only half of the sections $(54.40 \%)$ show access of the impression material deeper than the half of the gingival sulcus. The significant difference in the degree of the C-silicone's ingress of $12 \%$ is found between both studied chemical agents in favor of Xylometazolin $(\mathrm{p}<0.001)$. This fact highlights $0.05 \%$ Xylometazolin as a better retraction agent when applied with polydimethylsiloxane impression material. 
Iveta Katreva, Metodi Abadjiev, Simeon Simeonov et al.

As a result of the detailed analysis of the data collected during the study an obvious tendency can be noticed of more effective reversible displacement of soft tissues when the retraction cords are presoaked into $0.05 \%$ xylometazoline hydrochloride. Although the significant difference is found between Xylometazolin and Visine both $\alpha$-adrenomimetic substances can be applied successfully for the purposes of prosthetic dentistry.

\section{CONCLUSION}

a-adrenomimetic decongestants are effective alternative retraction agents for chemo-mechanical dilatation of the gingival groove. The promising data of the present and many more studies should change the term for these substances from "experimental" to "conventional" for their excellent hemostasis without cytotoxic alternation of periodontal tissues, harmful effect over hard tooth tissues and any risk for the overall health of patients.

\section{REFERENCES}

1. Kisov HK. Otpechatachni materiali i otpechatachni metodi v nepodvizhnoto zaboprotezirane. Sofia: Index; 1998. Bulgarian.

2. Abadzhiev M. Comparative research of the subgingival impression quality by fixed prosthesis using one and double cord retraction technique. Journal of IMAB - Annual Proceeding (Scientific Papers). 2009;2:52-54.

3. A Malbaker. Gingival Retraction - Techinques and Materials: A Review. Pakistan Oral \& Dental Journal. 2010;30(2).

4. Aimjirakul P, Masuda T, Takahashi H, Miura H. Gingival sulcus simulation model for evaluating the penetration characteristics of elastomeric impression materials. Int J Prosthodont. 2003;16:385-389.

5. Al-Ani A, Bennani V, Chandler NP, Lyons KM, Tomson WM. New Zealand dentists' use of gingival retraction techniques for fixed prosthodontics and implants. New Zeal Dent J. 2010;106:92-96.

6. Bowles WH, Tardy SJ, Vahadi A. Evaluation of the new gingival retraction agents. J Dent Res. 1991;70(11):1447-49.

7. Csillag M, Nyiri G, Vag J, Fazekas A. Dose-related effects of epinephrine on human gingival blood flow and crevicular fluid production used as a soaking solution for chemoch-mechanical tissue retraction. J Prosthed Dent. 2007;97(1):6-11.

8. Donovan TE, Chee WW. Current concepts in gingival displacement. Dent Clin North Am. 2004;48(2):433-44.

9. Felpel LP. A review of pharmacotherapeutics for prosthetic dentistry: Part I. J. Prosthet. Dent. 1997;3:285-292.

10. Hansen PA, Tira DE, Barlow J. Current methods of finish-line exposure by practicing prosthodontists. J. Prosthodont. 1999;8(3):163-70.

11. HE Strassler, L Boksman. Tissue Management, Gingival Retraction and Hemostasis. Available from: http://www.oralhealthgroup.com/news/ tissue-management-gingival-retraction-andhemostasis/1000519731/?\&er=NA

12. Kopač I, Batista U, Cvetko E, Marion L. Viability of fibroblasts in cell culture after treatment with different chemical retraction agents. J. Oral Rehab. 2002:29:98-104.

13. Liu C, Huang F, Yang L, Chou L, Chou M, Chanh Y. Cytotoxic effects of gingival retraction cords on human gingival fibroblasts in vitro. J. Oral Rehab. 2004;31:368-372.

14. Nowakowska $D$. The impact of retraction astringents on gingival margin tissues from literature review of in vivo studies. Protet. Stomatol. 2009;59:119-124.

15. Nowakowska D, Saczko J, Kulbacka J, Choromanska A. Dynamic oxidoreductive potential of astringent retraction agents. Folia Biol. (Praha). 2010;56:263-268.

16. Nowakowska D, Saczko J, Kulbacka J, Choromanska A, Raszewski Z. Cytotoxic potential of Vasoconstrictor Experimental Gingival Retraction Agents in Vitro Study on Primary Human Gingival Fibroblasts. Folia Biologica (Praha). 2012;58:37-43.

17. Pelzner RB, Kempler D, Stark MM, Lum LB, Nicholson RJ, Soelberg KB. Human blood pressure and pulse rate response to racemic epinephrine retraction cord. J Prosthed Dent 1978;39(3):287-92.

18. Strassler HE, Tissue Management, Gingival Retraction and Hemostasis. Benco Dental, Education Department, Available from: http://d3e9u3gw8odyw8. cloudfront.net/ie2_ce_tissue_management.pdf

Scripta Scientifica Medicinae Dentalis, vol. 1, №2, 2015, pp. 17-23 